\title{
Urokinase-type plasminogen activator receptor (UPAR), tissue factor (TF) and epidermal growth factor receptor (EGFR): tumor expression patterns and prognostic value in oral cancer
}

\author{
Anders Christensen ${ }^{12^{*}}$ D, Katalin Kiss ${ }^{3}$, Giedrius Lelkaitis ${ }^{3}$, Karina Juhl², Morten Persson², \\ Birgitte Wittenborg Charabi ${ }^{1}$, Jann Mortensen ${ }^{2}$, Julie Lyng Forman ${ }^{4}$, Anne Lyngholm Sørensen ${ }^{4}$, \\ David Hebbelstrup Jensen ${ }^{1}$, Andreas Kjaer ${ }^{2}$ \\ and Christian von Buchwald ${ }^{1}$
}

\begin{abstract}
Background: Tumor-specific biomarkers are a prerequisite for the development of targeted imaging and therapy in oral squamous cell carcinoma (OSCC). urokinase-type Plasminogen Activator Receptor (UPAR), Tissue Factor (TF) and Epidermal Growth Factor Receptor (EGFR) are three biomarkers that exhibit enhanced expression in many types of cancers, and have been investigated as potential biomarkers for targeted strategies and prognostication. The aim of the study was to investigate the expression patterns of UPAR, TF and EGFR and their potential prognostic value in OSCC.
\end{abstract}

Methods: Immunohistochemical expression of UPAR, TF and EGFR in tumor resection specimens from 191 patients with primary OSCC was analyzed. Overall (OS) and disease-free survival (DFS) was calculated. Associations between biomarker expression, clinicopathological factors and patient survival was analyzed using the Cox proportional hazards model for univariate and multivariate analysis, log rank and Kaplan-Meier statistics.

Results: UPAR and TF exhibited a highly tumor-specific expression pattern while EGFR also showed expression in normal tissues outside the tumor compartment. The overall positive expression rate of UPAR, TF and EGFR was 95\%, 58\% and $98 \%$, respectively. High uPAR expression across the entire cohort was negatively associated with OS $(p=0.031, \mathrm{HR}=1$. 595 (95\%Cl 1.044-2.439)) in univariate analysis. The 5-year OS for high and low UPAR expression was 39\% and 56\%, respectively. The expression of TF and EGFR was not associated with survival outcome.

Conclusions: This study may suggest that UPAR and TF could potentially be attractive targets for molecular imaging and therapy in OSCC due to high positive expression rates and tumor-specific expression patterns. High UPAR expression was significantly associated with a reduced survival. UPAR seems to be a prognostic biomarker in oral cancer.

Keywords: Oral squamous cell carcinoma, UPAR, Tissue factor, EGFR, prognosis, immunohistochemistry, margins, oral cancer, molecular imaging

\footnotetext{
* Correspondence: anders.christensen.03@regionh.dk

'Department of Otolaryngology, Head \& Neck Surgery and Audiology,

Rigshospitalet, University of Copenhagen, Copenhagen, Denmark

2Department of Clinical Physiology, Nuclear Medicine \& PET and Cluster for

Molecular Imaging, Rigshospitalet, University of Copenhagen, Copenhagen,

Denmark

Full list of author information is available at the end of the article
} 


\section{Background}

Oral cavity cancer is the 11th most common cancer worldwide and accounts for substantial mortality and morbidity for individuals affected by this disease [1]. Despite important technological advances in diagnosis and therapy especially in the last decades, the prognosis for OSCC has only moderately improved, and reported overall survival rate has remained at roughly 50\% [2]. Surgery is a cornerstone in the treatment of primary OSCC with curative intent, whether the objective is to achieve complete removal of the tumor as well as any regional metastatic disease in the neck. Failure to achieve a clear tumor-resection margin, and to detect residual disease in the surgical bed intraoperatively, has direct major negative impact on the chances for cure not fully compensated for by adjuvant radiotherapy [3, 4]. Intraoperative detection and delineation of cancer is still based on visual inspection and palpation of the tissues obviating reliable assessment of the microscopic extent of the disease. Consequently, non-radical surgery remains a major challenge, and novel imaging technology, that enables accurate planning of surgery and intraoperative tumor detection, is warranted.

The discovery of a large number of tumor-specific biomarkers has stimulated new optimism in the development of targeted imaging and treatment of cancer [5]. Ideally, a biomarker suitable for targeting purposes should have strong expression within the tumor compartment, and absent or insignificant expression in adjacent normal tissue. The expression of a specific biomarker may vary between different types of cancer, and within each specific type of cancer due to tumor heterogeneity, and therefore studies designed to examine the exact histological expression and tumor-specificity of different targets in large patient cohorts, are becoming increasingly important. Furthermore, accumulating evidence has validated biomarker expression and profiling as an important tool for individual risk stratification and planning of patient-tailored treatment [6]. The combined use of a specific biomarker as a prognosticator and a tumor-specific target for theranostic purposes is a novel strategy, which may have potential applications in the development of effective anti-cancer therapy. This study examined specifically the expression of UPAR and TF because our group has developed imaging and treatment agents targeting these to cell membrane receptors [7-9]. UPAR and TF have consistently been associated with cancer in most types of solid carcinomas [10, 11]. In addition, EGFR expression was investigated because it is an established target for therapy in HNSCC. However, data on the utility of EGFR as target for imaging agents are lacking.

In head and neck squamous cell carcinoma (HNSCC), the role of Endothelial Growth Factor Receptor (EGFR) in cancer progression has been extensively investigated. Several studies found EGFR overexpression to be a negative prognostic factor for local control and survival outcome measures for tumors arising in different subsites in the upper aerodigastive tract. However, existing data with regard to the prognostic role of EGFR in OSSC is ambiguous [12]. The recent clinical introduction of anti-EGFR agents (i.e. Cetuximab) for treatment of advanced HNSCC has emphasized the potential of EGFR as a target for anti-cancer therapy [13]. Importantly, a clinical trial on EGFR-targeted intraoperative optical tumor imaging was recently published, and EGFR-directed PET-imaging has been demonstrated in preclinical studies [14, 15].

UPAR signaling stimulates pericellular proteolysis facilitating plasmin-mediated extracellular matrix (ECM) degradation and subsequent tumor cell migration and invasion. Because of abundant implications in the carcinogenesis of numerous types of cancer, uPAR has been regarded as a promising biomarker for targeted molecular imaging and therapy [16-18]. Also in OSCC the pathophysiological role of the plasminogen activator system has been investigated, and UPAR has been appointed a key role in process of local invasion in the interplay with other cancer-associated proteolytic systems and signaling pathways [19]. However, there is a need to further uncover the prognostic value of UPAR in OSCC and to explore the rational of uPAR-targeted strategies in this cancer entity.

A strong relation between cancer and hemostasis is generally accepted, and aberrant venous thromboembolism is a common manifestation in malignant disease, including HNSCC $[20,21]$. TF is a transmembrane protein receptor and the principle initiator of the extrinsic coagulation cascade leading to fibrin formation after activation by its natural ligand factor VII. In addition TF activation has been associated with angiogenesis, tumor growth and invasion through regulation of the proteolytic cascade necessary for ECM degradation and tissue remodeling [22]. To our knowledge, TF expression in HNSCC has not been explored previously.

Accordingly, the aim of this study was to investigate the prognostic value and tumor expression patterns of EGFR, uPAR and TF in OSCC [23].

\section{Methods \\ Patients}

A cohort of 191 patients with primary OSCC, who underwent surgical tumor resection at the department of ORL - Head \& Neck Surgery \& Audiology at Rigshospitalet from 2000 to 2012, was retrospectively assembled. Inclusion criteria were primary OSCC in the mobile tongue or floor of mouth (FOM) with resection specimens available for immunohistochemical (IHC) 
analysis. Exclusion criteria were a previous history of HNSCC or radiotherapy to the head and neck region. Clinicopathological data were collected from medical records and pathology reports. All patients underwent clinical examination and radiological work-up and were staged at time of diagnosis according to the TNM classification by UICC, 7th edition [24]. Presence of regional nodal disease was determined based on pathology postoperatively $(\mathrm{pN})$. A clear margin $(>5 \mathrm{~mm})$ was defined, according to the Royal College of Pathologists, as the absence of involved $(<1 \mathrm{~mm})$ or close margins $(1-5 \mathrm{~mm})$ on routine histology [25]. If intraoperative frozen section technique was applied, absence of tumors cells in these specimens also defined a clear margin. For survival analysis the last day for follow-up was 16th August 2016, and time of surgery, time of death of all causes and time of recurrence was recorded. The study was approved by the Ethical Committee of the Capital Region of Denmark (protocol H-2-2012-050).

\section{IHC staining}

From formalin-fixed, paraffin-embedded tumor resection specimens, adjacent $4 \mu \mathrm{m}$ sections were prepared. IHC staining was performed on a semi-automated autostainer (Benchmark Ultra, Ventana- Roche, CA, USA). All antibodies were applied in optimized dilutions previously determined using positive and negative control staining. Briefly, slides were deparaffinized and rehydrated using EZ prep solution (Ventana-Roche, CA, USA). Following monoclonal antibodies were used: Cytokeratin (CK) clone AE1/AE3 (1:200, DAKO, Glostrup, Denmark), EGFR (RTU, Ventana-Roche, CA, USA), mouse anti-human uPAR R2 (1:20.000, Finsen Laboratory, Copenhagen, Denmark) [26], TF \#4509 (1:150, American Diagnostica Inc., Stamford, CT, USA). Antibody incubation time was $32 \mathrm{~min}$. For EGFR, uPAR and TF and $24 \mathrm{~min}$. For TF. Antigen retrieval for UPAR was done with protease $\mathrm{K}$ (Ventana-Roche, CA, USA) for $8 \mathrm{~min}$ followed by heating at $100{ }^{\circ} \mathrm{C}$ with cell conditioning 1 (CC1, Ventana-Roche, CA, USA) buffer for $16 \mathrm{~min}$. For CK, TF and EGFR standard heat induced epitope retrieval $\left(32 \mathrm{~min}, 100{ }^{\circ} \mathrm{C}\right)$ in $\mathrm{CC} 1$ buffer was used. IHC stainings were counterstained with hematoxylin. In addition, a section stained for hematoxylin-eosin (HE) of each case was prepared.

\section{Histology scoring}

All cases were reviewed and scored by two specialized head and neck pathologists (GL and KK) blinded to the clinicopathological data. For each case, the presence of tumor and extent of the tumor-compartment in relation to surrounding normal tissue was evaluated on HE and CK sections. The expression of uPAR, TF and EFGR was scored for intensity (I-score) and proportion of IHC reactivity within the tumor compartment (P-score). Both the I-score and the Pscore were based on a 4-point system: $0-3+$ (none, weak, moderate strong and $0-10 \%, 11-50 \%, 51-75 \%$, 76-100\%, respectively). For the I- and P-score IHC reactivity was not subdivided into expression on tumor cells and stromal cells, but was evaluated together, to represent reaction in the whole tumorcompartment. To combine information of intensity and proportion, a combined score (PI-score) was formed by addition of the I- and P-score as proposed by Allred and colleges [27]. The PI-score formed a 7 point system with a semi-quantitative scale from 0 to 6. In addition, sections were evaluated for homogeneous IHC expression within the tumor-compartment (yes/no), and IHC positivity in dysplastic epithelium if present (yes/ no). Because this paper investigated two separate research questions, expression patterns in relation to biomarker utility for targeted strategies and biomarker prognostication, respectively, the PI-score was dichotomized in two ways. For the evaluation of expression rate of uPAR, TF and EGFR, a tumor was considered positive if the PI-score was $>1$. A dichotomization in positive vs. negative was chosen because the positive expression rate across a cohort of patients is a key figure to determine the utility of a biomarker for targeted imaging. In the analysis of the prognostic value of UPAR, TF and EGFR, the cut-off values to separate low and high expression was defined as IPS $<6$ for uPAR and EGFR and IPS $<3$ for TF based on the distribution of the score for each biomarker. Histology sections were scanned using Axio Scan.Z1 (Carl Zeiss, Jena, Germany) to create digital images.

\section{Statistical analysis}

Associations between biomarker expression and clinicopathological variables were analyzed by Pearson's chisquare test or Fisher's exact test for small numbers. Age differences were investigated using the two-sample ttest. Overall survival (OS) was defined as time from primary surgery to death due to any cause, and diseasefree survival (DFS) was defined as time from primary surgery to cancer relapse or death by any cause. Associations between biomarker expressions and survival outcomes were visualized in Kaplan-Meier plots using the log-rank test to assess significance of differences. Also, the Kaplan-Meier method was used to estimate 5-year survival estimates. Hazard ratios were estimated in univariate and multivariate Cox proportional hazards model adjusting for gender, age, tobacco history, T-site, margin status, T-stage, $\mathrm{N}$-stage, extracapsular spread (ECS), TNM stage and tumor differentiation. A $p$-value $<0.05$ was considered statistically significant. All data analysis was performed in the SAS software package (SAS Institute Inc., version 6.1, USA). 


\section{Results}

The retrospective cohort of 191 patients with primary OSCC had a male predominance (66\%), the median age at time of surgery was 59 years (range: 23-89 years). The median follow-up was 5.1 years (range: 0.115.9 years). Demographics and the clinicopathological variables are listed in Table 1. Overall, the anatomical location of the tumors was distributed almost equally as 98 FOM tumors and 93 tongue tumors. The majority of the patients $(86 \%)$ presented with early stage disease (S1-S2) and most tumors (90\%) were well differentiated (G1-G2). 31\% were diagnosed with primary regional nodal disease and 51 patients (27\%) had post-treatment relapse. For the entire cohort the 5-year OS and DFS was $51 \%$ and $41 \%$, respectively.

\section{Histopathological biomarker expression patterns}

Based on the examination of the immunohistochemical staining in resection specimens, UPAR and TF were found to be highly tumor-specific, with enhanced expression within the tumor compartment, and absent or very limited expression in the normal tissues surrounding the tumors (Figs. 1 and 2). EGFR was less tumorspecific due to regular expression in normal tissues. The overall positive expression rate of UPAR, TF and EGFR was $95 \%, 58 \%$ and $98 \%$, respectively. High expression of uPAR, TF and EGFR was observed in 28\%, 39\% and 40\% of the tumors, respectively (Table 2).

uPAR was expressed on neoplastic cells as well as on fibroblasts and inflammatory cells in the tumor compartment. In addition, stromal expression of UPAR was also the predominant pattern. The same pattern was observed in isolated clusters of neoplastic cells invading into the stroma (Fig. 2). Also, in the majority of the tumors, strong uPAR expression was found in a narrow and well demarcated peritumoral reactive zone at the invasive front. uPAR expression in dysplastic epithelium adjacent to the carcinoma was very limited (4.1\%, Table 2 ). In most specimens, solitary uPAR-positive neutrophils were observed in the tumor compartment as well as in normal tissues. In two cases, strong uPAR staining was noted on macrophages and neutrophils in an abscess located outside the tumor compartment.

Within the tumor compartment, TF was predominantly expressed in a heterogeneous pattern on tumor cells, and intense staining was generally noted at the invasive edge of the tumors (Fig. 2). TF expression in dysplastic epithelium was noted in some cases (16.3\%) and the basal cells of normal epithelium showed weak TF-positivity in all cases. Generally, a weak staining of TF was observed in the collecting ducts in salivary gland tissue outside the tumor compartment.

EGFR was found to have highly homogeneous expression on neoplastic cells in the majority of the tumors
(Table 2). Moreover, EGFR expression outside the tumor compartment was observed in the normal epithelium in all cases. In specimens containing dysplasia, EGFR was expressed in the dysplastic epithelium in $93.9 \%$ of the cases. In adjacent normal salivary gland tissue, regular EGFR expression was seen on the epithelial cells (Fig. 1).

\section{Correlation analysis of biomarker expression and clinicopathological parameters}

Associations between biomarker expression and clinicopathological variables are summarized in Table 1. High uPAR expression was significantly correlated with an advanced TNM stage $(p=0.022)$. High TF expression was significantly associated with tumor location in the tongue $(p=0.018)$ and relapse of disease $(p=0.001)$. No other significant associations between the three biomarkers and clinicopathological variables were observed.

\section{Survival analysis}

Univariate and multivariate survival analysis using the Cox proportional hazard model for OS and DFS with respect to variables are summarized in Table 3. In OS and DFS 126 and 177 events were recorded, respectively. Kaplan Meier curves combined with log rank analysis for differences showed a significant association between high UPAR expression in tumors and OS but not DFS, and no significant correlations was found for TF and EGFR (Fig. 3). The 5-year OS was 55.5\% for low uPAR expression and $39.1 \%$ for high uPAR expression $(p=0.030)$. High uPAR expression showed a significant negative association with $\mathrm{OS}$ in the univariate analysis $(p=0.031, \mathrm{HR}=1.595(95 \% \mathrm{CI} 1.044-2.439))$ but significance of this association was not retained in the multivariate analysis $(p=0.128$, HR $=1.435$ (95\%CI $0.901-$ 2.287)). uPAR expression did not reach statistical significance in DFS. For TF and EGFR no significant association with survival outcome was detected. To investigate a possible prognostic value of UPAR, TF and EGFR in early low-risk disease, univariate and multivariate analysis was also performed for the subgroups S1-S2 $(n=136)$, T1-T2 $(n=164)$ and G1-G2 $(N=171)$. High uPAR expression reached a significant association with OS only in the univariate analysis in all three subgroups but not in the multivariate analysis. However, in the subgroup of well differentiated tumors (G1-G2) a borderline significant association was detected in multivariate analysis $(p=0.051, \mathrm{HR}=1.618(95 \% \mathrm{CI} 0.997-2.625))$.

As expected, high T-stage, high disease stage, involved or close resection margins, primary nodal metastasis and presence of ECS was associated with poor outcome in OS and DFS in univariate analysis. Only margin status and $\mathrm{N}$-stage retained significance in multivariate analysis. Also increasing age was associated with reduced OS and DFS in univariate and multivariate analysis. 


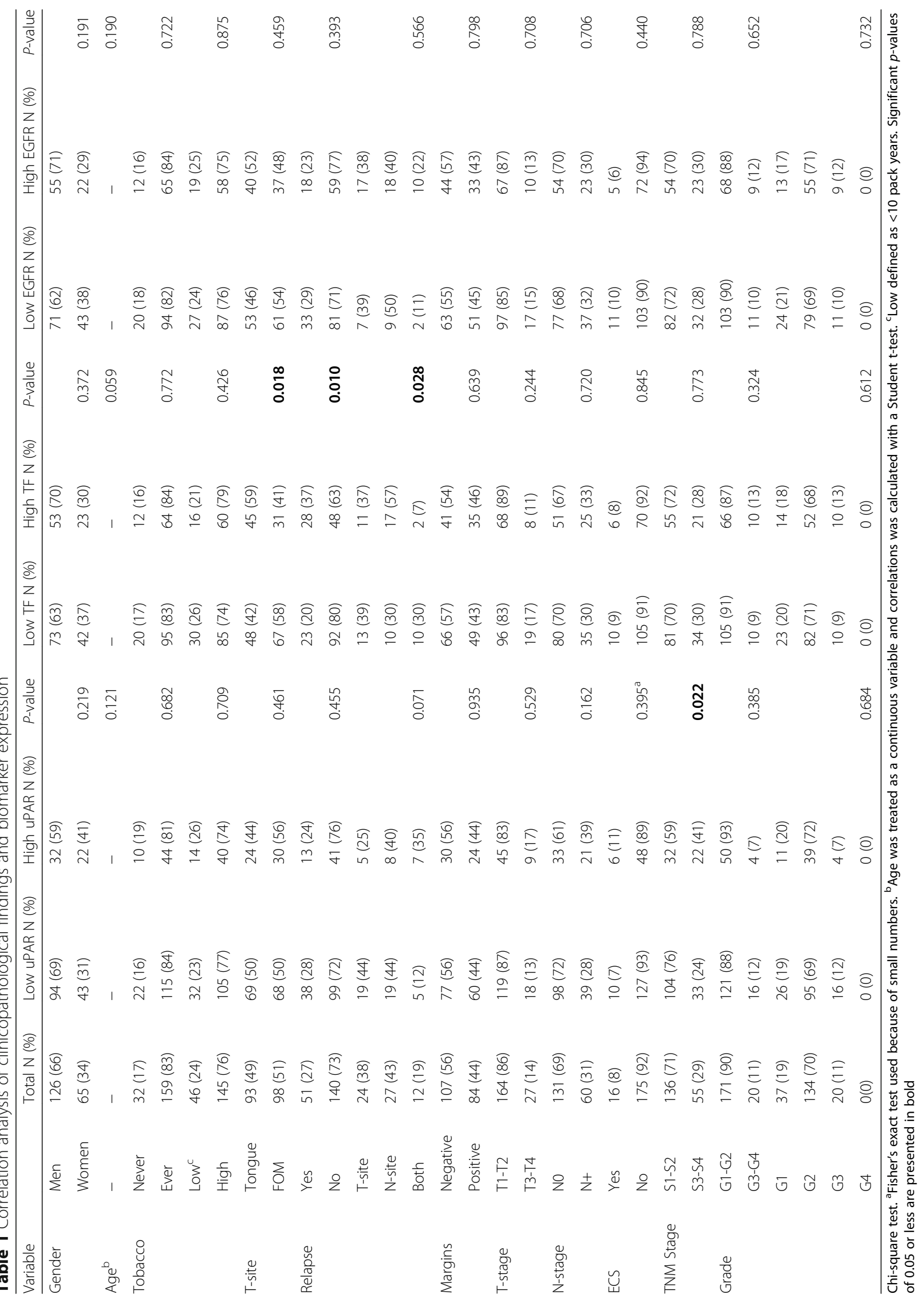



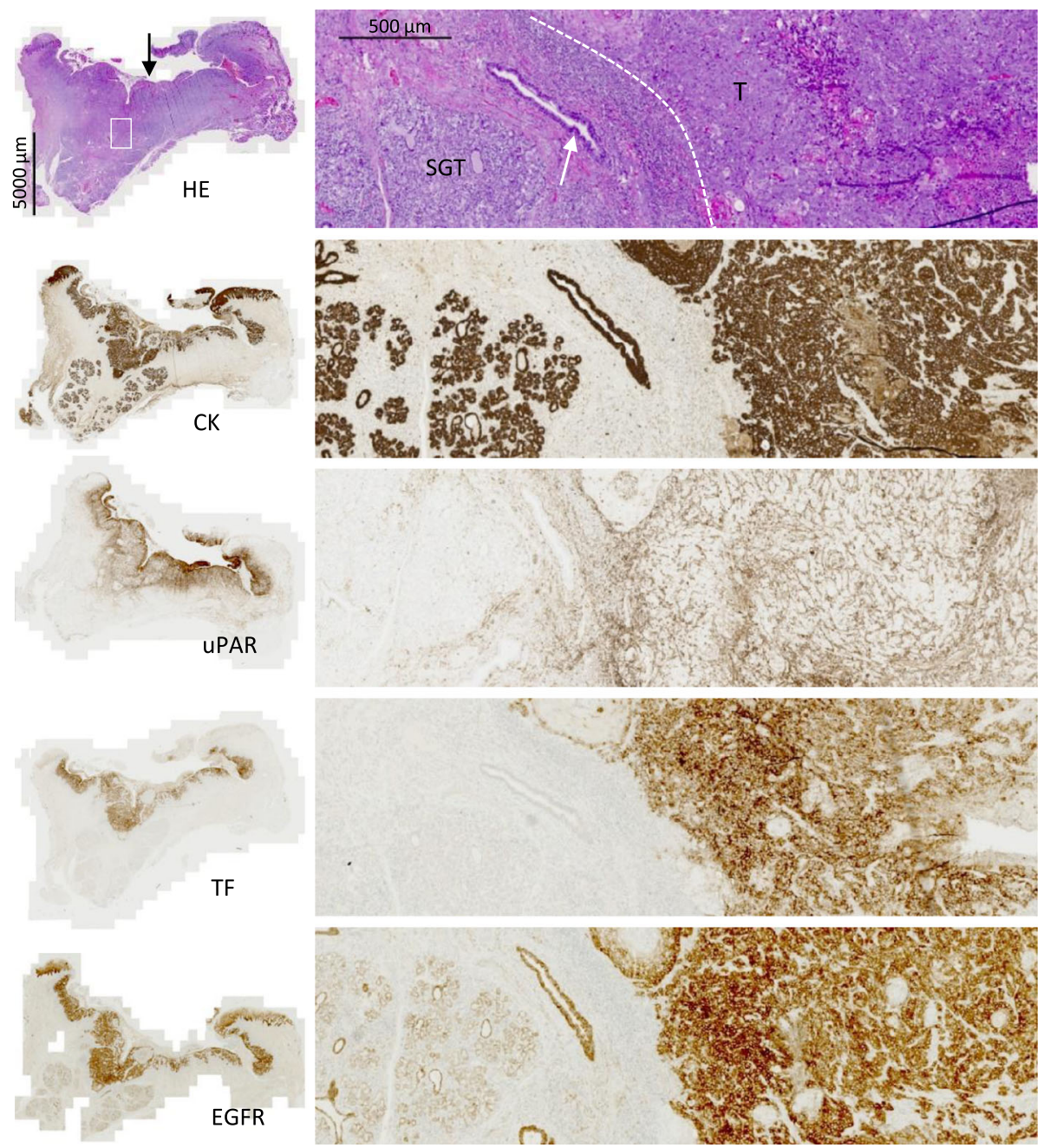

Fig. 1 Patterns of expression of UPAR, TF and EGFR. Adjacent tissue sections from a T1 FOM tumor (T). Black arrow indicates the epithelial lining in the oral cavity. White square shows the location of the enlarged region of interest presented to the right. Dotted white line shows the invasive front of the tumor. A large collecting salivary duct (white arrow) and salivary gland tissue (SGT) located adjacent to the tumor border. EGFR expression is noted on neoplastic cells as well as in the epithelium of salivary gland tissue

\section{Discussion}

This study provides novel data compared to existing literature because it was based on IHC biomarker expression in whole tumor resection specimens of OSCC and not only biopsy specimens. Resection specimens containing both the tumor compartment and the adjacent resection margin of healthy tissue is a prerequisite to accurately examine the utility of a biomarker for tumorspecific molecular targeting. Also, resection specimens allows to assess the heterogeneity of the biomarker expression across the tumor compartment as opposed to studies based on biopsies that only sample a very small fraction of the tumor. To our knowledge, this type of study of UPAR and TF expression in OSCC has not previously been reported.

UPAR and TF both showed an enhanced expression specifically confined to the tumor compartment with very limited expression in the normal tissues surrounding the neoplasm. In contrast, EGFR lacked a tumorspecific expression pattern and therefore the ability to distinguish between malignant and normal tissues. Further, the positive expression rate of uPAR and TF was high, which implies, that a substantial part of OSCC patients should be regarded as candidates for imaging and/or therapy directed against either uPAR or TF. Especially uPAR was found to have a highly tumorspecific pattern, and also with very limited expression in both normal and dysplastic epithelium around the epithelial tumor lesion. In comparison, EGFR exhibited staining of both normal and dysplastic epithelium in most cases, and importantly also a general EGFR expression in salivary gland tissue outside the tumor compartment was seen. A prerequisite to develop highly accurate targeted imaging is that the molecular target 
a
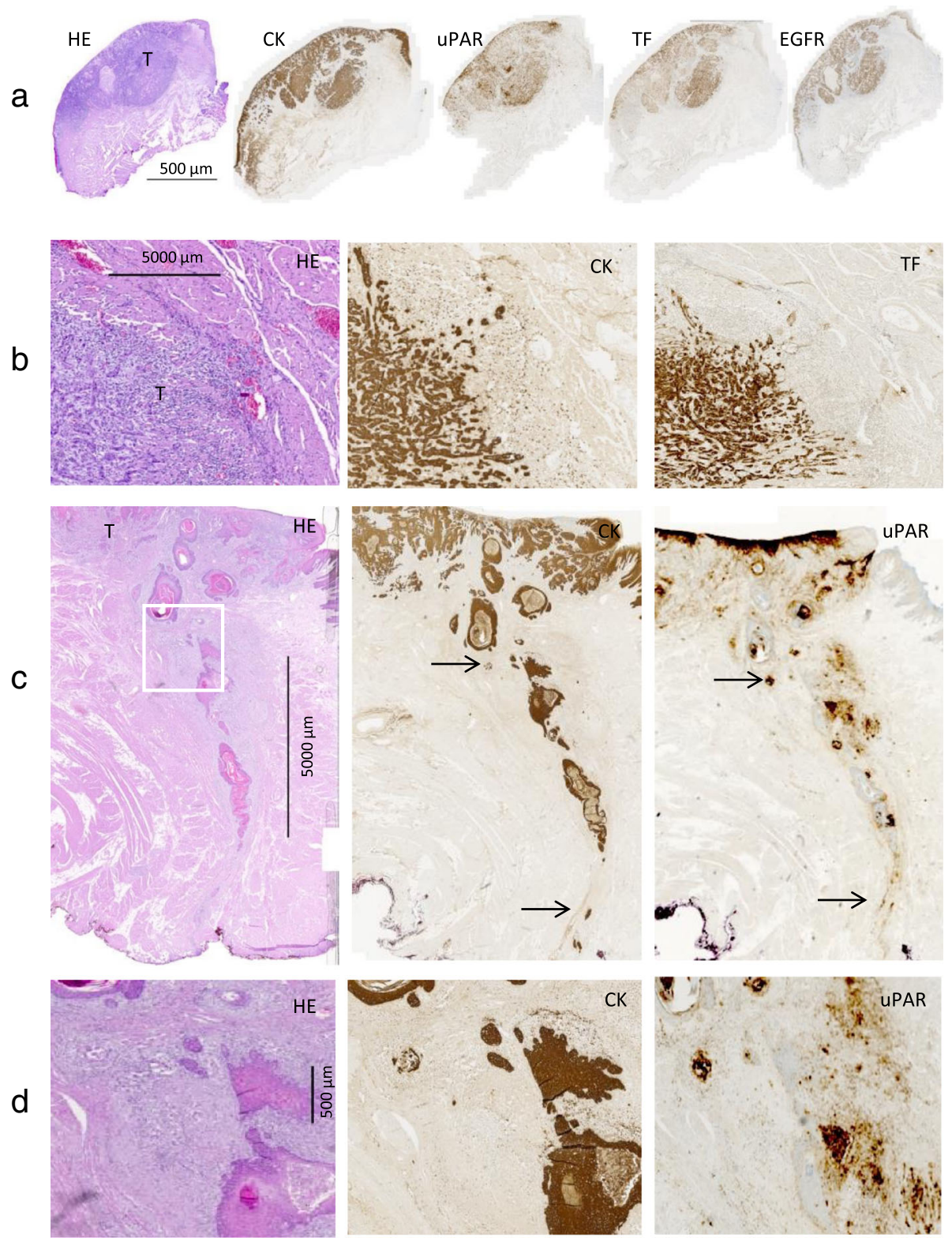

Fig. 2 Selected features of biomarker expression. a T2 tongue SCC (T). Expression of UPAR, TF and EGFR confined to the tumor compartment. $\mathbf{b}$ An example of strong TF expression on the neoplastic cells at the invasive front of a tongue SCC (T). c An example of a tongue SCC T) invading deeply into the underlying stroma. Black arrows indicate expression of CK and UPAR on small tumor cell groups and the white square shows the location of the enlarged region of interest depicted in panel (d)

exhibits very low expression in normal tissues bordering the tumor to create a high tumor-to-normal tissue ratio. Targeted optical-guided surgery is currently being clinically translated, and this imaging modality will allow intraoperative real-time assessment of resection margins in order to ensure complete removal of tumors. Further, our group and others have recently presented preclinical data derived from animal models of oral cancer, showing that detection of subclinical disease by use of targeted fluorescent probes is possible $[28,29]$. However, because an optical imaging signal has a low energy with a limited range in intensity, target binding of an imaging agent outside the tumor compartment in normal tissues would potentially have substantial influence on the ability to detect a reliable tumor-specific signal to guide a tumor resection. Accordingly, in the data from the recently published first phase 1 trial of targeted optical imaging in HNSCC, using the optical agent cetuximab-IRDye800 directed against EGFR, extratumoral signal uptake in normal epithelium and salivary gland tissue in tissue sections was reported [30]. 
Table 2 Expression patterns of UPAR, TF and EGFR in OSCC

\begin{tabular}{lllll}
\hline Variable & & UPARN (\%) & TFN (\%) & EGFRN (\%) \\
\hline Positive biomarker expression & Yes & $182(95)$ & $110(58)$ & $188(98)$ \\
& No & $9(5)$ & $81(42)$ & $3(2)$ \\
Homogeneous expression & Yes & $107(56)$ & $50(26)$ & $163(85)$ \\
in the tumor compartment & No & $84(44)$ & $141(74)$ & $28(15)$ \\
Expression in dysplastic $_{\text {epithelium }^{\text {a }}}$ & Yes & $4(4)$ & $16(16)$ & $92(94)$ \\
& No & $94(96)$ & $82(84)$ & $6(6)$ \\
\hline
\end{tabular}

${ }^{a}$ Dysplastic epithelium was present in 98 out of 191 tumor samples

Direct comparison of the positive expression rates of uPAR, TF and EGFR in this study and previous studies is not possible because of difference in scoring systems used and because of different cut-off values to determine positive and negative expression. Three studies investigated expression of UPAR in OSCC and reported a positive expression rate in the range of $39-100 \%$ [31-33]. Positive EGFR expression in OSCC was in the range of $60-100 \%$ in previous studies [34-37]. We found a positive expression rate of $58 \%$ for TF, but the expression of TF in OSCC has not previously been investigated. Chen et al. found a TF immunopositivity of $91 \%$ in esophageal cancer [38].

To select potential biomarkers for targeted imaging, van Oosten et al. suggested a selection criteria tool identifying seven factors on order of importance: (1) Extracellular receptor location, (2) diffuse enhanced target expression in tumor compartment, (3) high tumor-tonormal tissue target expression, (4) high expression-rate in patients, (5) previous successful targeted imaging in vivo, (6) enzymatic activity of the receptor and (7) target receptor internalization [39]. In relation to the results of the present study, both uPAR, TF and EGFR fulfills the factors $1-5$, which underlines the relevance of these receptors for targeted tumor imaging in OSCC. In addition, UPAR is also reported to exhibit enzymatic activity in the tumor microenvironment and internalization to the intracellular space upon ligand-binding, which makes this receptor especially suited for targeting by imaging agents [11].

In the correlation analysis, high TF expression was associated with relapse of disease. However, TF expression did not show significant impact on OS or DFS in the survival analysis, which has been reported in colorectal, breast and esophageal cancer [38, 40, 41]. We found high UPAR expression to be associated with late TNM stage disease (S3-S4). However, no significant association was reached between UPAR and $\mathrm{N}$ - or T-stage analyzed separately. In a study of 115 patients with OSCC, Magnusson et al. reported that low expression of uPAR was correlated with reduced disease specific death only in patients with stage 1 (S1) disease [42]. Bacchiochi et al. analyzed the prognostic value of UPAR in 189 patients with OSCC and found enhanced UPAR expression to be associated with increasing tumor cell differentiation, and that low uPAR expression only was associated with increased OS in well differentiated tumors [31]. Our study confirms the findings in the latter study, as high uPAR expression only for the sub-group of well differentiated tumors was associated negatively with OS in the univariate analysis and reached borderline significance in the multivariate analysis. In two studies from the same Japanese group, containing 34 and 54 OSCC patients, respectively, high uPAR expression was associated with an aggressive mode of invasion [43, 44]. Unfortunately, our study did not include the pattern of invasion (cohesive vs. non-cohesive invasive tumor front) as a histopathological variable, but further research in the relation between local tumor aggressiveness and UPAR activity in OSCC is warranted.

In the survival analysis in the present study, enhanced uPAR expression was associated with a significant reduction in OS only, while no significant associations between expression of TF or EGFR and survival outcome could be demonstrated. Accordingly, our data supports findings in previous studies, that uPAR expression seems to be a prognostic factor for survival outcome in OSCC [31, 42, 44]. However, we did not find uPAR to be an independent prognostic factor in multivariate analysis. Also, uPAR was associated with advanced TNM stage. Therefore, UPAR expression could also be a surrogate for advanced stage of disease as well as comorbidity. A larger sample size would be able to clarify the meaning of these findings.

Interestingly, our study is the first to show that uPAR had impact on survival outcome across an entire cohort of patients, and not only in a subgroup analysis of patients defined by a specific clinicopathological variable $[31,42]$ or a combination of biomarker expressions [44]. We did not find any significant association between EGFR expression and survival outcome, which is consistent with several previous studies, although some studies have associated enhanced EGFR expression with poor clinical outcome [12]. Of note, a limitation of this study was a relatively small sample size with a limited number of outcome events, which may have affected the ability to detect significant correlations between biomarker expression and survival. In addition, assessment of biomarker expression based on immunohistochemistry has an inherent limited accuracy due to intra-observer variability and the design of the scoring systems used.

Consistent with a study of Lindberg et al., we also found that UPAR predominantly was expressed on stromal cells of the tumor compartment [33]. Similar findings have been reported in colorectal and esophageal cancer [45, 46]. Stroma-rich tumors, like OSCC, may be less sensitive to targeted therapy, if the target 


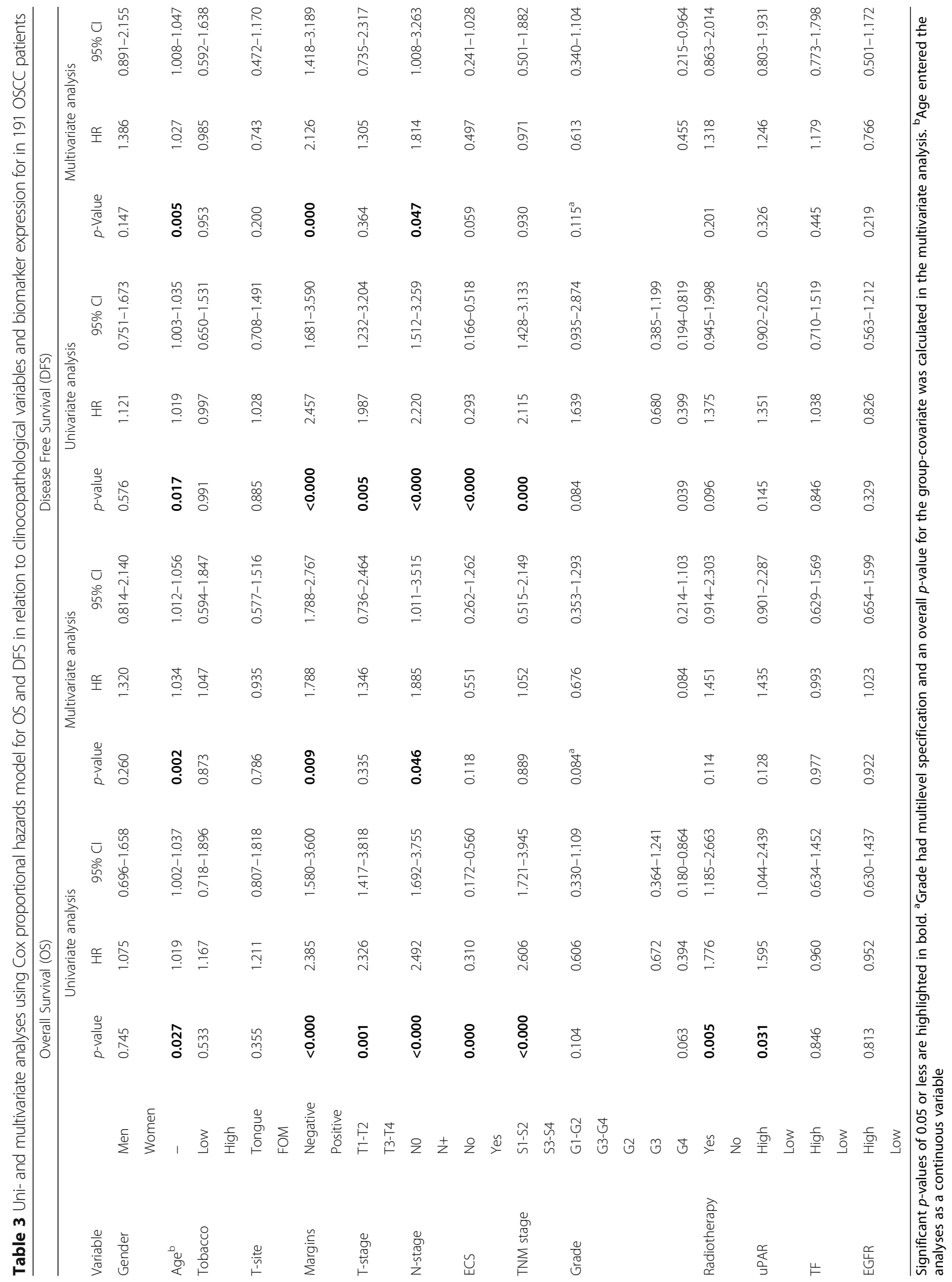




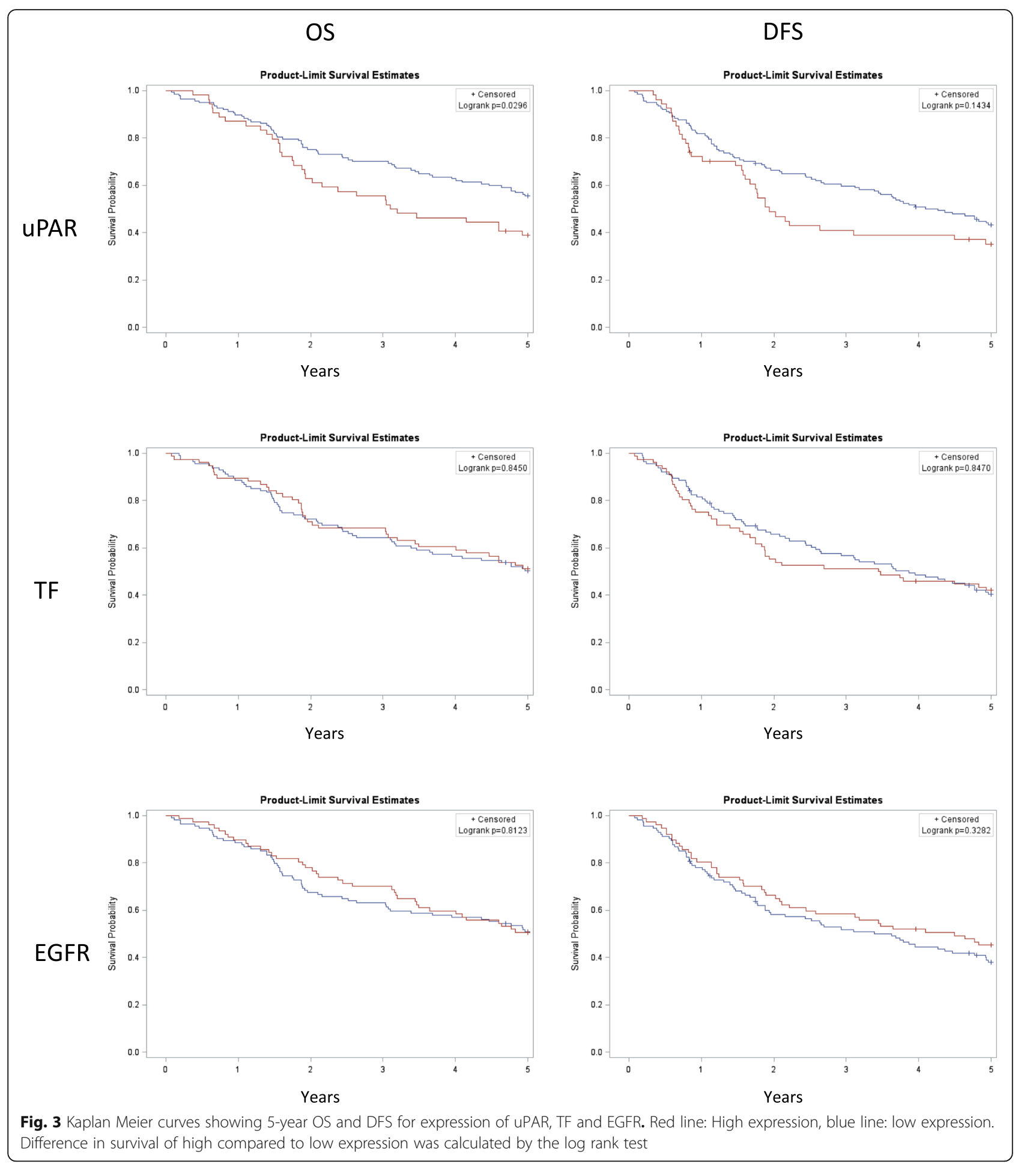

only is expressed by tumor cells, because of reduced target density in the tumor compartment. Therefore, uPAR expression on both tumor cells and tumorassociated stromal cells may provide uniform target availability in the entire tumor volume. This expression pattern may be a particular advantage in order to achieve high efficacy of uPAR-targeted intervention and therapy.

Existing data indicate, that elevated uPAR expression in OSCC and several other types of cancer within the tumor seem to predict a more aggressive phenotype that carries reduced survival outcome [18]. Therefore UPAR 
may have a role to play as a reliable prognostic biomarker in future personalized management of OSCC. Based on the favorable properties of UPAR as an imaging target, a clinical phase 2 trial of preoperative uPAR-PET/CT imaging in patients with oral or oropharyngeal SCC is currently being conducted in our institution (NCT02960724). uPAR-PET imaging may provide a non-invasive quantitative assessment of the UPAR expression in the entire volume of individual tumors, thereby surpassing the inherent problems related to surgical biopsies and risk of sampling error due to tumor heterogeneity.

\section{Conclusions}

Our results showed that both UPAR and TF had high positive expression rates and tumor-specific expression patterns while EGFR also had regular expression in normal tissues. These findings may suggest that uPAR and TF could potentially be attractive targets for molecular imaging and targeted therapy in OSCC.

High uPAR expression was significantly associated with reduced survival outcome. Accordingly, uPAR seems to be a potential prognostic biomarker in OSCC, which may have applications for risk-stratification and treatment planning.

\section{Abbreviations}

95\%Cl: 95\% Confidence Interval; CC1: Cell conditioning 1; DFS: Disease-free Survival; EGFR: Epidermal Growth Factor Receptor; FOM: Floor of mouth; HNSCC: Head \& Neck Squamous Cell Carcinoma; IHC: Immunohistochemistry; OS: Overall Survival; OSCC: Oral Squamous Cell Carcinoma; TF: Tissue Factor; UPAR: Urokinase-type Plasminogen Activator Receptor

\section{Acknowledgements}

The authors thank Pernille Frederiksen, in the Department of Pathology for technical laboratory assistance.

\section{Funding}

This paper was supported by University of Copenhagen, the Agnes \& Poul Friis Foundation and the Harboe Foundation and the three funds supported costs related to laboratory analyses and statistical interpretation of data.

\section{Availability of data and materials}

The dataset analyzed during this study is available from the corresponding author on reasonable request.

\section{Authors' contributions}

AC designed to overall study in collaboration with $K K, G L, K J, M P, B W C, J M$, $\mathrm{DHJ}, \mathrm{AK}$ and $\mathrm{CvB}$. AC and DHJ collected specimens and organized the cohort. KK and GL supervised the immunohistochemistry and conducted the histopathological scoring. AC, JLF and ALS performed and interpreted the statistical data analysis. AC drafted the manuscript and KK, GL, K, MP, BWC, JM, JLF, ALS, DHJ, AK and CvB critically reviewed and finalized the manuscript before submission. All authors have approved the final version of this manuscript.

\section{Ethics approval and consent to participate}

Approval for this study was obtained from the Ethical Committee of the Capital Region of Denmark (protocol H-2-2012-050). Data was anonymized prior to analysis. The Ethical Committee waived from the need to obtain consent to participate from the patients due to the circumstance that a great part of the cohort had died or were severely ill, and that information about this project would lead to more distress than good for the patients.

\section{Consent for publication}

Not applicable.

\section{Competing interests}

The authors declare that they have no competing interest.

\section{Publisher's Note}

Springer Nature remains neutral with regard to jurisdictional claims in published maps and institutional affiliations.

\section{Author details}

${ }^{1}$ Department of Otolaryngology, Head \& Neck Surgery and Audiology, Righhospitalet, University of Copenhagen, Copenhagen, Denmark.

${ }^{2}$ Department of Clinical Physiology, Nuclear Medicine \& PET and Cluster for Molecular Imaging, Rigshospitalet, University of Copenhagen, Copenhagen, Denmark. ${ }^{3}$ Department of Pathology, Rigshospitalet, University of Copenhagen, Copenhagen, Denmark. ${ }^{4}$ Department of Public Health, Section of Biostatistics, University of Copenhagen, Copenhagen, Denmark.

Received: 15 February 2017 Accepted: 17 August 2017

Published online: 25 August 2017

\section{References}

1. Fitzmaurice C, Dicker D, Pain A, Hamavid H, Moradi-Lakeh M, Maclntyre MF, et al. The Global Burden of Cancer 2013. JAMA Oncol. 2015;1 (4):505-27.

2. Argiris A, Karamouzis MV, Raben D, Ferris RL. Head and neck cancer. Lancet. 2008:371(9625):1695-709.

3. Bradley PJ, MacLennan K, Brakenhoff RH, Leemans CR. Status of primary tumour surgical margins in squamous head and neck cancer: prognostic implications. Curr Opin Otolaryngol Head Neck Surg. 2007;15(2):74-81.

4. Haque R, Contreras R, McNicoll MP, Eckberg EC, Petitti DB. Surgical margins and survival after head and neck cancer surgery. BMC Ear Nose Throat Disord. 2006;6:2

5. Dahiya K, Dhankhar R. Updated overview of current biomarkers in head and neck carcinoma. World J Methodol. 2016;6(1):77-86.

6. Mes SW, Leemans CR, Brakenhoff RH. Applications of molecular diagnostics for personalized treatment of head and neck cancer: state of the art. Expert Rev Mol Diagn. 2016:16(2):205-21.

7. Nielsen CH, Erlandsson M, Jeppesen TE, Jensen MM, Kristensen LK, Madsen J, et al. Quantitative PET Imaging of Tissue Factor Expression Using 18FLabeled Active Site-Inhibited Factor VII. J Nucl Med. 2016;57(1):89-95.

8. Nielsen $\mathrm{CH}$, Jeppesen TE, Kristensen LK, Jensen MM, El Ali HH, Madsen J, et al. PET Imaging of Tissue Factor in Pancreatic Cancer Using 64Cu-Labeled Active Site-Inhibited Factor VII. J Nucl Med. 2016;57(7):1112-9.

9. Skovgaard D, Persson M, Brandt-Larsen M, Christensen C, Madsen J, Klausen TL, et al. Safety, Dosimetry, and Tumor Detection Ability of 68Ga-NOTAAE105: First-in-Human Study of a Novel Radioligand for UPAR PET Imaging. J Nucl Med. 2017;58(3):379-86.

10. van den Berg YW, Osanto S, Reitsma PH, Versteeg HH. The relationship between tissue factor and cancer progression: insights from bench and bedside. Blood. 2012;119(4):924-32.

11. Noh H, Hong S, Huang S. Role of urokinase receptor in tumor progression and development. Theranostics. 2013;3(7):487-95.

12. Bossi P, Resteghini C, Paielli N, Licitra L, Pilotti S, Perrone F. Prognostic and predictive value of EGFR in head and neck squamous cell carcinoma. Oncotarget. 2016:7(45):74362-79.

13. Trivedi S, Concha-Benavente F, Srivastava RM, Jie HB, Gibson SP, Schmitt NC, et al. Immune biomarkers of anti-EGFR monoclonal antibody therapy. Ann Oncol. 2015;26(1):40-7.

14. Rosenthal EL, Warram JM, de Boer E, Chung TK, Korb ML, Brandwein-Gensler M, et al. Safety and Tumor Specificity of Cetuximab-IRDye800 for Surgical Navigation in Head and Neck Cancer. Clin Can Res. 2015;21(16):3658-66.

15. van Dijk LK, Boerman OC, Kaanders JH, Bussink J. Epidermal growth factor receptor imaging in human head and neck cancer xenografts. Acta Oncol. 2015;54(9):1263-7.

16. Persson M, Rasmussen P, Madsen J, Ploug M, Kjaer A. New peptide receptor radionuclide therapy of invasive cancer cells: in vivo studies using 177LuDOTA-AE105 targeting UPAR in human colorectal cancer xenografts. Nucl Med Biol. 2012;39(7):962-9.

17. Persson M, Juhl K, Rasmussen P, Brandt-Larsen M, Madsen J, Ploug M, et al. UPAR targeted radionuclide therapy with (177) Lu-DOTA-AE105 inhibits dissemination of metastatic prostate cancer. Mol Pharm. 2014;11(8):2796-806. 
18. Persson M, Kjaer A. Urokinase-type plasminogen activator receptor (UPAR) as a promising new imaging target: potential clinical applications. Clin Physiol Funct Imaging. 2013;33(5):329-37.

19. Shi Z, Stack MS. Urinary-type plasminogen activator (UPA) and its receptor (UPAR) in squamous cell carcinoma of the oral cavity. Biochem J. 2007; 407(2):153-9.

20. Clayburgh DR, Stott W, Cordiero T, Park R, Detwiller K, Buniel M, et al. Prospective study of venous thromboembolism in patients with head and neck cancer after surgery. JAMA otolaryngol Head Neck Surg. 2013;139(11):1143-50.

21. Yapijakis C, Bramos A, Nixon AM, Ragos V, Vairaktaris E. The interplay between hemostasis and malignancy: the oral cancer paradigm. Anticancer Res. 2012;32(5):1791-800.

22. Han X, Guo B, Li Y, Zhu B. Tissue factor in tumor microenvironment: a systematic review. J Hematol Oncol. 2014;7:54.

23. 6th World Congress International Academy of Oral Oncology (IAOO). Head Neck. 2017;39 Suppl 1:E83.

24. Sobin S, Gospodarowicz M, Wittekind C. TNM Classification of Malignant tumours - Seventh Edition. Wiley-Blackwell; 2009.

25. Woolgar JA, Triantafyllou A. A histopathological appraisal of surgical margins in oral and oropharyngeal cancer resection specimens. Oral Oncol. 2005:41(10):1034-43.

26. Ronne E, Behrendt N, Ellis V, Ploug M, Dano K, Hoyer-Hansen G. Cellinduced potentiation of the plasminogen activation system is abolished by a monoclonal antibody that recognizes the $\mathrm{NH} 2$ - terminal domain of the urokinase receptor. FEBS Lett. 1991;288(1-2):233-6.

27. Allred DC, Harvey JM, Berardo M, Clark GM. Prognostic and predictive factors in breast cancer by immunohistochemical analysis. Mod Pathol. 1998;11(2):155-68.

28. Christensen A, Juhl K, Persson M, Charabi BW, Mortensen J, Kiss K, et al. UPAR-targeted optical near- infrared (NIR) fluorescence imaging and PET for image-guided surgery in head and neck cancer: proof- of-concept in orthotopic xenograft model. Oncotarget. 2016;

29. Atallah I, Milet C, Henry M, Josserand V, Reyt E, Coll JL, et al. Near-infrared fluorescence imaging guided surgery improves recurrence-free survival rate in novel orthotopic animal model of head and neck squamous cell carcinoma. Head Neck. 2016;38(Suppl 1):E246-55

30. de Boer E, Warram JM, Tucker MD, Hartman YE, Moore LS, de Jong JS, et al. In Vivo Fluorescence Immunohistochemistry: Localization of Fluorescently Labeled Cetuximab in Squamous Cell Carcinomas. Sci Rep. 2015:5:10169.

31. Bacchiocchi R, Rubini C, Pierpaoli E, Borghetti G, Procacci P, Nocini PF, et al. Prognostic value analysis of urokinase-type plasminogen activator receptor in oral squamous cell carcinoma: an immunohistochemical study. BMC Cancer. 2008:8:220.

32. Curino A, Patel V, Nielsen BS, Iskander AJ, Ensley JF, Yoo GH, et al. Detection of plasminogen activators in oral cancer by laser capture microdissection combined with zymography. Oral Oncol. 2004;40(10):1026-32.

33. Lindberg P, Larsson A, Nielsen BS. Expression of plasminogen activator inhibitor-1, urokinase receptor and laminin gamma-2 chain is an early coordinated event in incipient oral squamous cell carcinoma. Int J Cancer. 2006;118(12):2948-56

34. Chung CH, Ely K, McGavran L, Varella-Garcia M, Parker J, Parker N, et al. Increased epidermal growth factor receptor gene copy number is associated with poor prognosis in head and neck squamous cell carcinomas. J Clin Oncol. 2006:24(25):4170-6.

35. Smid EJ, Stoter TR, Bloemena E, Lafleur MV, Leemans CR, van der Waal I, et al. The importance of immunohistochemical expression of EGFr in squamous cell carcinoma of the oral cavity treated with surgery and postoperative radiotherapy. Int J Radiat Oncol Biol Phys. 2006;65(5):1323-9.

36. Dassonville O, Formento JL, Francoual M, Ramaioli A, Santini J, Schneider M, et al. Expression of epidermal growth factor receptor and survival in upper aerodigestive tract cancer. J Clin Oncol. 1993;11(10):1873-8.

37. Chandarana SP, Lee JS, Chanowski EJ, Sacco AG, Bradford CR, Wolf GT, et al. Prevalence and predictive role of p16 and epidermal growth factor receptor in surgically treated oropharyngeal and oral cavity cancer. Head Neck. 2013; 35(8):1083-90.

38. Chen L, Luo G, Tan Y, Wei J, Wu C, Zheng L, et al. Immunolocalisation of tissue factor in esophageal cancer is correlated with intratumoral angiogenesis and prognosis of the patient. Acta Histochem. 2010;112(3):233-9.

39. van Oosten M, Crane LM, Bart J, van Leeuwen FW, van Dam GM. Selecting Potential Targetable Biomarkers for Imaging Purposes in Colorectal Cancer Using TArget Selection Criteria (TASC): A Novel Target Identification Tool. Transl Oncol. 2011;4(2):71-82.
40. Shigemori C, Wada H, Matsumoto K, Shiku H, Nakamura S, Suzuki H. Tissue factor expression and metastatic potential of colorectal cancer. Thromb Haemost. 1998;80(6):894-8.

41. Ueno $T$, Toi $M$, Koike $M$, Nakamura $S$, Tominaga $T$. Tissue factor expression in breast cancer tissues: its correlation with prognosis and plasma concentration. Br J Cancer. 2000;83(2):164-70.

42. Magnussen S, Rikardsen OG, Hadler-Olsen E, Uhlin-Hansen L, Steigen SE, Svineng G. Urokinase plasminogen activator receptor (UPAR) and plasminogen activator inhibitor-1 (PAl-1) are potential predictive biomarkers in early stage oral squamous cell carcinomas (OSCC). PLoS One. 2014;9(7):e101895.

43. Nozaki S, Endo Y, Kawashiri S, Nakagawa K, Yamamoto E, Yonemura Y, et al. Immunohistochemical localization of a urokinase-type plasminogen activator system in squamous cell carcinoma of the oral cavity: association with mode of invasion and lymph node metastasis. Oral Oncol. 1998;34(1):58-62.

44. Yoshizawa K, Nozaki S, Kitahara H, Kato K, Noguchi N, Kawashiri S, et al. Expression of urokinase-type plasminogen activator/urokinase-type plasminogen activator receptor and maspin in oral squamous cell carcinoma: Association with mode of invasion and clinicopathological factors. Oncol Rep. 2011;26(6):1555-60.

45. Boonstra MC, Verbeek FP, Mazar AP, Prevoo HA, Kuppen PJ, van de Velde CJ, et al. Expression of UPAR in tumor-associated stromal cells is associated with colorectal cancer patient prognosis: a TMA study. BMC Cancer. 2014;14:269

46. Laerum OD, Ovrebo K, Skarstein A, Christensen IJ, Alpizar-Alpizar W, Helgeland $L$, et al. Prognosis in adenocarcinomas of lower oesophagus, gastro-oesophageal junction and cardia evaluated by UPAR immunohistochemistry. Int J Cancer. 2012;131(3):558-69.

\section{Submit your next manuscript to BioMed Central and we will help you at every step:}

- We accept pre-submission inquiries

- Our selector tool helps you to find the most relevant journal

- We provide round the clock customer support

- Convenient online submission

- Thorough peer review

- Inclusion in PubMed and all major indexing services

- Maximum visibility for your research

Submit your manuscript at www.biomedcentral.com/submit
) Biomed Central 